\title{
Un itinéraire antique entre Corre (Haute-Saône) et Escles (Vosges) confirmé par le LiDAR : vers la reconnaissance de voies de portage entre la Saône et
} la Moselle

\author{
Ancient itinerary between Corre (Haute-Saône) and Escles (Vosges) \\ confirmed by the LiDAR: towards the recognition of portage ways \\ between the Saône and the Moselle
}

\author{
Pierre Fetet ${ }^{1}$ \\ ${ }^{1}$ Docteur en histoire ancienne, Escles-Archéologie, pfetet@free.fr
}

RÉSUMÉ. Les couvertures LiDAR sous forêt permettent d'identifier des habitats ruraux mais également des voies de circulation, locales ou de plus grand parcours. Depuis 1989, l'association Escles-Archéologie organise des prospections et répertorie les voies antiques de la Vôge, région forestière et rurale située au sud-ouest du département des Vosges. L'une d'elles attire particulièrement lattention car ses substructions la rendent facilement repérable au sol. On la considère comme une voie de portage entre la Saône et la Moselle, l'agglomération gallo-romaine d'Escles pouvant être une étape à mi-chemin entre les deux rivières. En 2016, l'étude d'un relevé LiDAR a permis de révéler une bande de terre de 40 mètres de large, bordée par des fossés rectilignes, qui emprunte le même tracé que la voie au nord de la Saône. Cette bande de terre fait partie d'un axe général rectiligne de 27 kilomètres de Corre à Escles avec un azimut de $30^{\circ} \mathrm{E}$. Au franchissement des vallées, alors que la voie pavée s'écarte pour trouver des pentes adoucies, la bande de terrain conserve son orientation rectiligne jusqu'à la rupture de pente en bordure du plateau, puis franchit l'obstacle grâce à des rampes très pentues et reprend sur l'autre rive la même largeur et la même direction. Les relevés effectués sur la section Corre-Escles permettent aujourd'hui de connaître assez précisément l'orientation et le tracé de ces deux cheminements contigus et de confirmer l'existence d'une voie de portage rectiligne importante destinée probablement aux piétons, aux cavaliers et aux bêtes de somme. La voie pavée, quant à elle, devait être utilisée pour transporter des charges lourdes avec des charriots. Cette étude, qui révèle la complexité topographique et chronologique des liaisons routières entre la Saône et la Moselle, appelle de nouvelles campagnes de prospections, de fouilles et de relevés LiDAR pour préciser les phasages et les tracés, en particulier pour la section Escles-Portieux qui est peu documentée jusqu'à présent.

ABSTRACT. LiDAR covers under forest allow to identify rural settlements and roads. Since 1989, the association EsclesArchéologie has been organizing surveys and listing the ancient ways of the Vôge. One of them attracts particular attention because its substructures make it easy to identify on the ground. It is thought to have been a portage route between the Saône and the Moselle; the Gallo-Roman village of Escles may have been a mid-way stopover between the two rivers. In 2016, the examination of a LiDAR survey revealed a strip of land 40 meters wide, bordered by straight ditches, which follows the road to the north of the Saône. This strip of land is part of a 27 kilometers straight line from Corre to Escles with an azimuth of $30^{\circ} \mathrm{E}$. When crossing the valleys, while the road deviates to follow easier slopes, this strip of land retains its rectilinear orientation until the change in the gradient at the edge of the plateau, then crosses the obstacle by very steep ramps and resumes the same width and direction on the other side of the river. Surveys carried out on the Corre-Escles section now make it possible to know quite precisely the orientation and the layout of these two contiguous paths and to confirm the existence of a large rectilinear portage way probably intended for pedestrians, riders and pack animals. On the other hand, the paved way was to be used to carry heavy loads. This study, which reveals the topographical and chronological complexity of the road connections between the Saone and the Moselle, requires new archaeological investigations and LiDAR surveys to clarify the routes, in particular for the Escles-Portieux section which is so far poorly documented.

MOTS-CLÉS. voie, portage, LiDAR, itinéraire, navigation.

KEYWORDS. way, portage, LiDAR, route, navigation. 


\section{Historique et contexte des recherches}

L'association Escles-Archéologie organise des prospections pédestres et répertorie, entre autres, les voies antiques de la Vôge (sud-ouest du département des Vosges). L'une d'elles a particulièrement attiré l'attention des prospecteurs car ses substructions - des blocs de grès mis de chant - la rendent facilement repérable au sol dès que l'on est sous couvert forestier. Cette voie avait été signalée sommairement dès le $\mathrm{XIX}^{\mathrm{e}}$ siècle par Charles-Louis Mangin ; cet érudit local la considérait comme une voie reliant la Saône à la Moselle (Mangin 1821). Malgré la légitimité de son hypothèse, elle ne fut reprise par aucun historien. On sait pourtant par Strabon que les principaux cours d'eau de la Gaule étaient non seulement navigables mais aussi reliés par des routes. L'écrivain explique dans sa Géographie que les cours d'eau assuraient, dans les deux sens, les transports d'une mer à l'autre et que les marchandises pouvaient être transportées par voie de terre sur des distances courtes, «toujours dans des plaines d'une traversée facile ${ }^{1} \gg$. En ce qui concerne la voie terrestre reliant la Saône à la Moselle, voie dont l'existence est mentionnée par Tacite, l'itinéraire était connu pour être plutôt difficile ${ }^{2}$. En effet, le franchissement de la ligne de partage des eaux entre les deux rivières nécessitait de traverser un relief, la Vôge, dénommé les monts Vosegus dans l'Antiquité3, qui, découpé par de nombreuses vallées encaissées, s’apparentait plus à une montagne qu'à une plaine.

\section{Présentation générale du relief et des cours d'eau}

La Vôge est un plateau gréseux triasique qui, lors du soulèvement du massif vosgien, a basculé vers l'ouest, s'inclinant vers le bassin de la Saône. Sa topographie est marquée par la présence de plateaux entaillés de vallées profondes; elles suivent de grands accidents structuraux dont le rejet vertical peut atteindre par endroits plus de $200 \mathrm{~m}$ comme on peut le constater dans la vallée du Coney.

Si la quasi-totalité de la surface de la Vôge est drainée par des cours d'eau appartenant au bassin de la Saône, et dépend donc du Rhône et de la mer Méditerranée, le plateau est également entaillé au nord par la haute vallée du Madon qui entraîne les eaux vers la Moselle, le Rhin et la mer du Nord. Deux sources de rivières renommées, le Madon et la Saône, ne sont distantes que de $2 \mathrm{~km}$, toutes deux situées respectivement sur les territoires d'Escles et de Vioménil et séparées par la limite de partage des eaux qui culmine dans cette zone au Ménamont à $466 \mathrm{~m}$.

\section{Un itinéraire de transbordement de Corre à Portieux}

\subsection{L'état de la recherche avant 2016}

Avant de pouvoir étudier les données LiDAR de l'ONF, les prospections pédestres avaient permis de repérer une voie reliant Corre à Escles (Fetet 2009a). Proche de ces deux localités - considérées toutes deux comme des agglomérations gallo-romaines (Mangin \& Bonvallot 2002 et Bertin et al. 1999) - des tronçons d'une voie plus ou moins rectiligne ayant une orientation générale identique, à savoir un azimut proche de $30^{\circ} \mathrm{E}$, ont été reconnus sur le terrain. Au nord de Corre, cette direction est conservée sur au moins $4 \mathrm{~km}$ et au sud d'Escles sur $5 \mathrm{~km}$. Ces deux localités étant distantes de $27 \mathrm{~km}$ l'une de l'autre, un tiers de cet itinéraire était donc clairement identifié. En 2001, durant la fouille du sanctuaire de Mercure de la Pille (Vioménil, 88) ( $c f$. fig. 2, repère étoile), la structure de cette voie a pu être observée : la coupe a révélé une assise de pavés de grès de 20 à $30 \mathrm{~cm}$ de longueur posés de chant, bordés d'une rangée de blocs plus gros, et recouverts d'une couche très compacte de gravier, de galets et de terre argileuse d'une épaisseur de $50 \mathrm{~cm}$ comprenant quelques blocs de grès, le tout formant la

\footnotetext{
${ }^{1}$ Strabon, Géogr., IV, 1, 2.

${ }^{2}$ « sublatisque itineris difficultatibus » : Tacite, Ann., XIII, 53.

3 " Arar Germaniae fluvius e Vogeso monte miscetur Rhodano " : Vibius Sequester, De fluminibus, fontibus, lacubus quorum apud poetas fit mentio.
} 
dernière couche de roulement (Gaffiot 2001). Des éléments de substruction, pavés ou graviers, ont été reconnus à divers endroits de l'itinéraire : à Escles, les blocs de grès alignés apparaissent dans le ruisseau de Buissonrupt en deux strates également (Bertin et al. 1998). Plus au sud, ses fossés sont révélés par une photographie aérienne à Escles au lieu-dit Clochimont (Fetet 2009b, $2: 106$ ). Au bois Le Baulot (Escles), puis dans la forêt de la Pille (Vioménil), on peut suivre son terrassement ou son pavage rectiligne jusqu'au sanctuaire de Mercure (Bertin et al. 1997). À cet endroit, la fouille a permis de raccorder archéologiquement ce site gallo-romain à la voie grâce à la découverte d'un sentier pavé (Gaffiot 2005). De ce fait, il n'existe aucun doute sur sa datation antique. À $50 \mathrm{~m}$ en amont du sanctuaire, une voie se raccorde à la voie principale dans la direction du vallon de la Pille afin de traverser la vallée de la Saône en pente douce. Pourtant, la voie principale continue aussi de son côté à suivre son tracé rectiligne vers la Saône, mais on perd sa trace au niveau de la clairière de la Bataille. Ces deux tracés conjoints nous intriguaient car nous n'imaginions pas qu'une route, fût-elle antique, puisse descendre le côté abrupt de la rive droite de la vallée de la Saône à cet endroit particulièrement escarpé. Le relevé LiDAR nous donnera plus tard la solution (cf. § 3.2.).

Sur les deux coteaux de la Saône, entre la Pille et la Bataille, les traces archéologiques de la voie secondaire ne manquent pas car ses pavés sont visibles soit directement sous les feuilles mortes, soit à la faveur d'une coupe de terrain due à un fossé de chemin, une tranchée de drainage ou un ruisseau (Bertin et al. 2003). Après avoir rejoint le plateau sur la rive gauche de la Saône, la voie pavée se dirige vers Hennezel où un tronçon a été entièrement dégagé de 1986 à 1991 dans le canton de SainteMarie (fig. 1) (Bertin et al. 1997). Sa largeur est de $6 \mathrm{~m}$ à cet endroit contre un peu plus de $4 \mathrm{~m}$ au sanctuaire de la Pille. Comme pour la traversée du vallon de la Saône, la voie se dévie de l'axe idéal entre Escles et Corre à l'approche du vallon encaissé de l'Ourche. Plus au sud, son tracé devient incertain jusqu'au lieu-dit Thomas, puis on rencontre son tracé à la Croix du Gendarme sur le territoire de la commune de Passavant-la-Rochère. Ce n'est qu'au niveau du bois Barbey que l'on retrouve le même axe rectiligne que l'on suivait sur les finages d'Escles et de Vioménil, sous la forme d'un chemin séparant les territoires des communes de Vougécourt et Demangevelle. Cette route, dénommée à cet endroit «Voie de Lorraine », se poursuit jusqu'au village de Corre (Faure-Brac 2002 : 195).

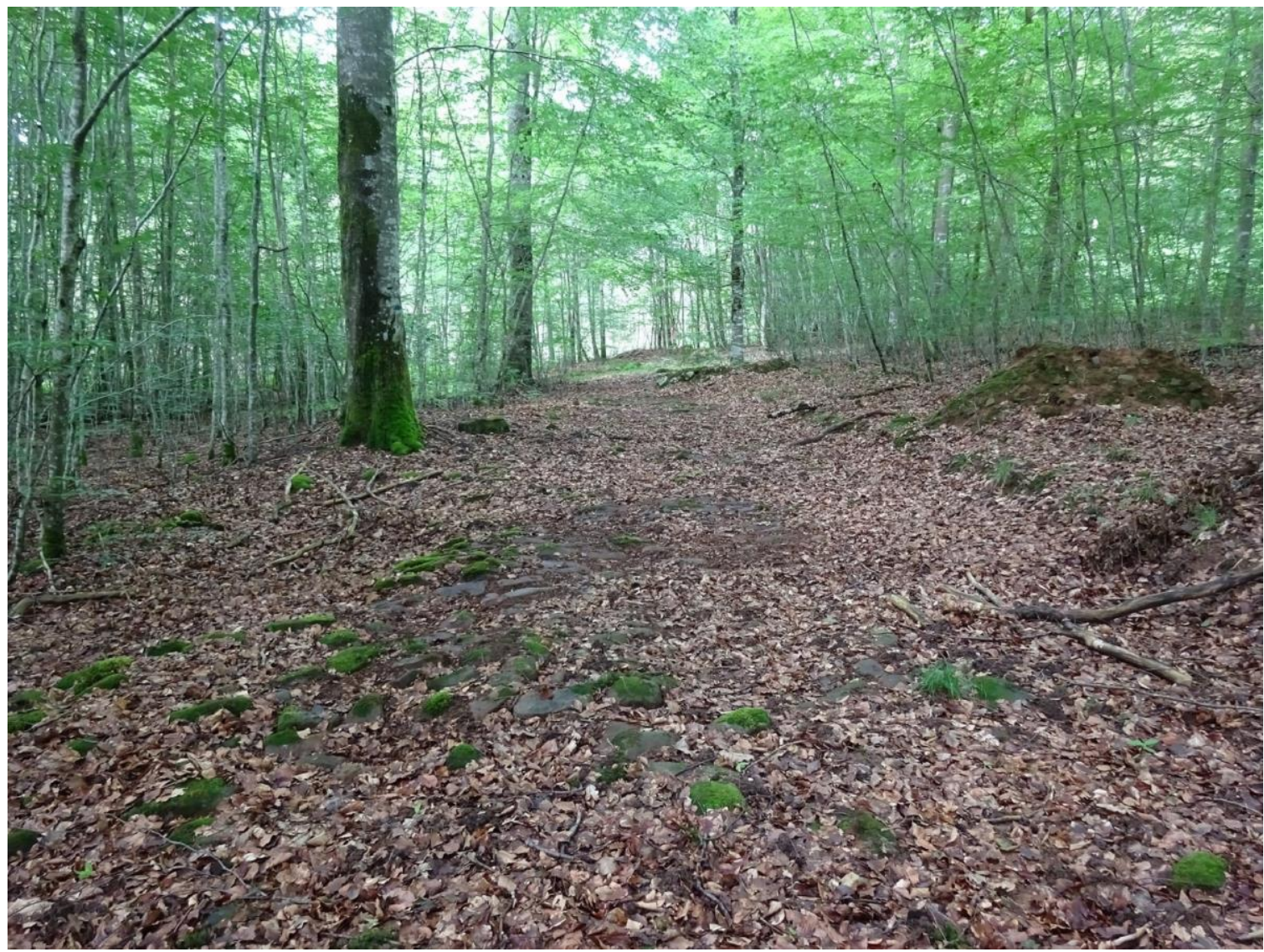

Fig. 1. Voie pavée d'Hennezel au canton de Sainte-Marie (Cliché P. Fetet) 
Au nord d'Escles, des prospections ont été entreprises pour retrouver cette voie qui pourrait rejoindre le gué de Portieux sur la Moselle (Bertin et al. 2001) où a été découverte au XIX ${ }^{\mathrm{e}}$ siècle une colonne de Jupiter à l'anguipède ${ }^{4}$ (fig. 2). Des textes d'archives et des indices cadastraux, toponymiques et physiques nous font penser que l'axe ne s'arrêtait pas à Escles mais l'occupation actuelle du territoire, plus dense, ne nous a pas permis pour l'instant d'en reconnaître des traces archéologiques assurées au sol. Les recherches récentes pour déterminer quel itinéraire serait idéal pour une voie de portage entre la Saône et la Moselle ayant un itinéraire court et bon marché privilégient également un axe Corre-Charmes (Pasquini \& Petit 2016), Charmes se situant à $5 \mathrm{~km}$ en aval de Portieux. De plus, des poutrages en chêne, qui peuvent être considérés comme des aménagements portuaires, ont été retrouvés à proximité des endroits théoriques de connexion de la voie aux deux rivières : à Portieux dans la berge droite de la Moselle (Bertin et al. 1999) et à Corre lors des travaux de creusement de la marina en 1995 (Fetet 2009b, $1: 158$ ).

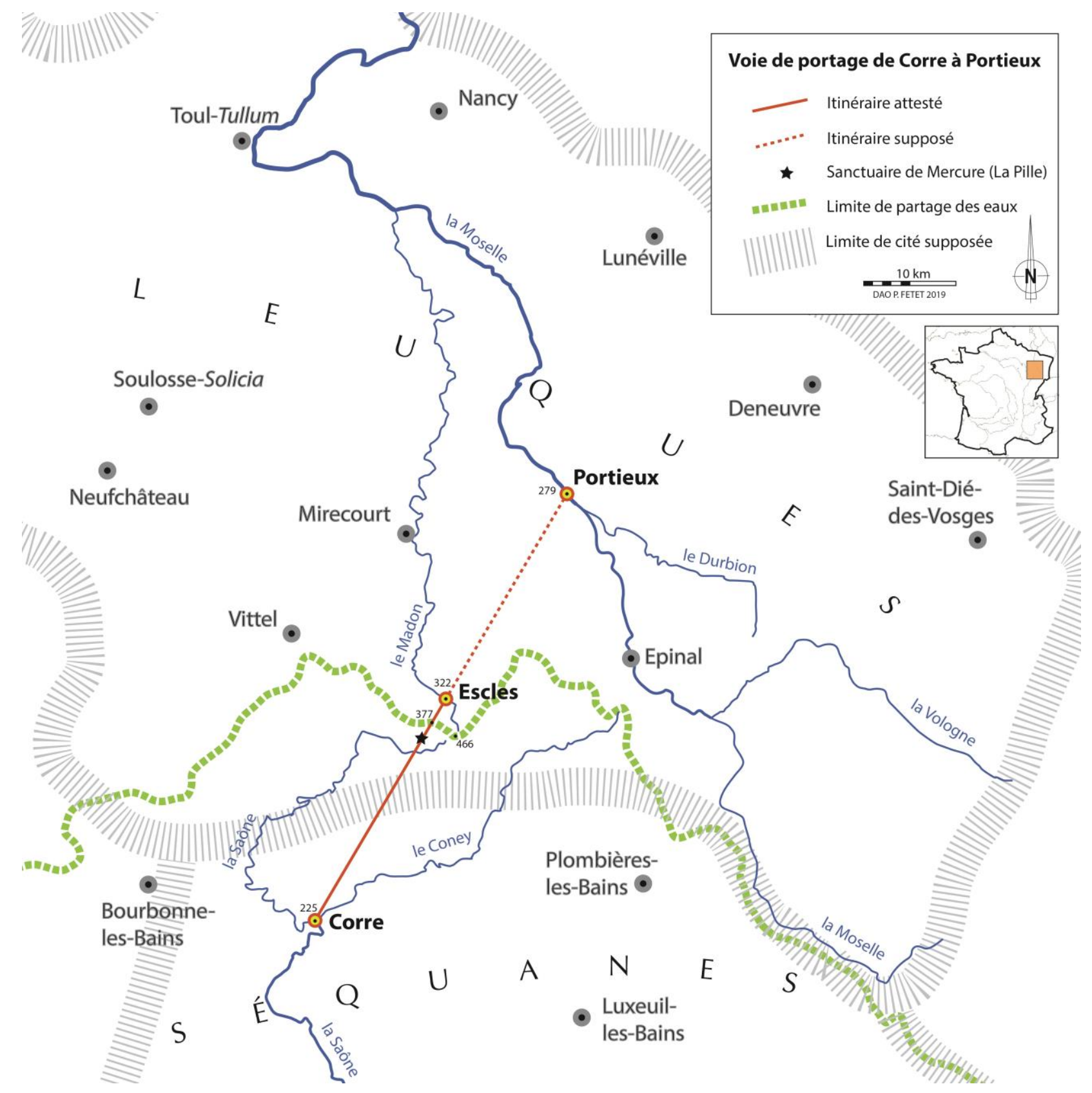

Fig. 2. Situation de l'axe Corre-Portieux via Escles pouvant correspondre à une voie de portage entre la Saône et la Moselle (DAO P. Fetet)

\footnotetext{
${ }^{4}$ Le cavalier à l'anguipède est exposé au Musée d'art ancien et contemporain d'Épinal. Des éléments d'une colonne au dieu cavalier ont également été découverts près du port antique de Corre (Kerrio 2019).
} 


\subsection{L'apport du LiDAR}

Les données du relevé LiDAR ont été enregistrées sur la forêt de Darney en mars 2014 en période hors feuille pour le compte de la Direction territoriale de la Lorraine de l'Office National des Forêts. La commande, confiée à la société Sintegra, a consisté en l'établissement d'un modèle numérique de terrain (MNT) et de canopée (MNCE) avec une densité de 1 point $/ \mathrm{m}^{2}$ et pour une surface de 16000 hectares. Le partenariat avec Escles-Archéologie a été rendu possible en 2016 grâce à Murielle Georges-Leroy, alors conservatrice régionale de l'archéologie de Lorraine. Le traitement des données permettant de ne conserver que les points de la surface du sol (MNT), la microtopographie a pu être analysée de manière assez fine en jouant sur l'orientation des éclairages rasants ${ }^{5}$, ce qui a révélé des reliefs invisibles en prospection de terrain. Pour ce qui concerne la voie de Corre à Escles, le LiDAR a ainsi permis d'une part de confirmer les connaissances acquises sur le terrain depuis les premières recherches et d'autre part d'apporter des informations jusqu'alors impossibles à visualiser. La principale découverte a été la mise en évidence d'une bande de terre large d'environ 40 mètres, délimitée par deux fossés rectilignes et se trouvant exactement sur l'axe idéal entre Corre et Escles à quelques degrés près. Les fossés repérés sont des microreliefs invisibles au sol qui ne sont visualisés que dans les forêts. Ailleurs, les défrichements et les labours ont effacé ce terrassement. Il est possible que cette délimitation de terrain corresponde à une trouée de défrichement antique correspondant à la traversée d'un massif forestier relativement important délimitant les territoires leuque et séquane ${ }^{6}$ (Fetet 2009b). Ces reliefs naturels ont servi également par la suite de frontière entre la Lorraine et la Franche-Comté et c'est bien leur caractère montagneux qui explique cette persistance en tant que limite. On peut donc dire que ce sont les pentes abruptes qui ont généré la frontière plus que l'espace forestier. Dans le cas où la Vôge était, comme aujourd'hui, un massif forestier au moment de la création de ces sillons rectilignes, leur existence n'a pu être possible qu'après un défrichement au minimum sur le tracé de ces lignes qui ont nécessité des visées. Il est probable que, dans cette hypothèse, le défrichement s'est poursuivi entre l'espace de ces fossés pour améliorer la visibilité du chemin mais il nous est impossible de le prouver dans l'état actuel de nos connaissances.

Nous allons décrire dans un premier temps cette bande de terre qui suit un tracé directeur rectiligne de Passavant-la-Rochère à Vioménil puis, dans un deuxième temps, les tronçons de voies qui lui sont jointes ou contiguës.

\subsubsection{Description de la bande de terre de Passavant-la-Rochère à Vioménil (fig. 3 et 4)}

La bande de $40 \mathrm{~m}$ de large environ n'est pas visible en continu mais par tronçons. Sa visualisation est interrompue par des vallées ou par des clairières établies par des verriers au Moyen Âge ou à l'Époque moderne. Quelquefois, une seule bordure est décelable, ou alors c'est un relief flou indiquant un terrassement rectiligne.

Entre Corre et Escles, les images générées à partir du LiDAR de Passavant-la-Rochère à Vioménil sont sans équivoque : un axe semble avoir été tracé au cordeau, aligné à la «voie de Lorraine » au sud et à la voie antique observée à Escles au nord. Si l'azimut général entre Corre et Escles est d'environ $30^{\circ} \mathrm{E}$, il varie légèrement de $1^{\circ}$ en plus ou en moins selon les tronçons.

Ce tracé rectiligne est coupé par trois vallées principales, celle de la Morte-Eau à Passavant-laRochère, celle de l'Ourche à Hennezel et celle de la Saône à Vioménil. Pour chacune de ces vallées, il est impossible pour un véhicule et quelquefois pour un piéton de rester sur l'axe directeur car les pentes sont trop abruptes ou le cheminement est arrêté par un escarpement rocheux. Les concepteurs de ce couloir rectiligne ont résolu ce problème en aménageant des pentes naturelles plus douces dans la

\footnotetext{
${ }^{5}$ Un ombrage unidirectionnel (azimut $315^{\circ}$, altitude $10^{\circ}$ ) a été appliqué au modèle numérique de terrain.

${ }^{6}$ Si l'on retient l'hypothèse que le terrain parcouru par la bande rectiligne était forestier, sa réalisation a nécessité le défrichement d'une surface considérable correspondant à plus d'une centaine d'hectares.
} 
continuité de l'axe ou en créant des rampes dans le versant. Le terrain oblige donc les constructeurs à adapter le tracé théorique sur de petites distances. Dès que le relief le permet à nouveau, la bande de terre reprend l'axe directeur général.

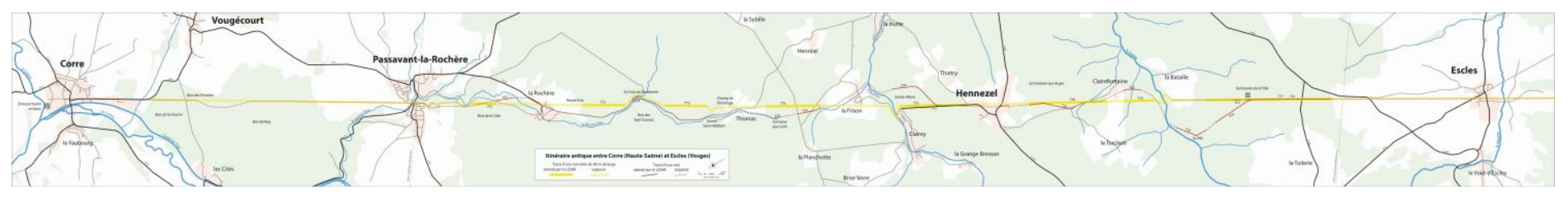

Fig. 3. Carte de l'itinéraire antique de Corre à Escles (DAO P. Fetet)

PROFIL ALTIMÉTRIQUE DE L'ITINÉRAIRE CORRE-ESCLES

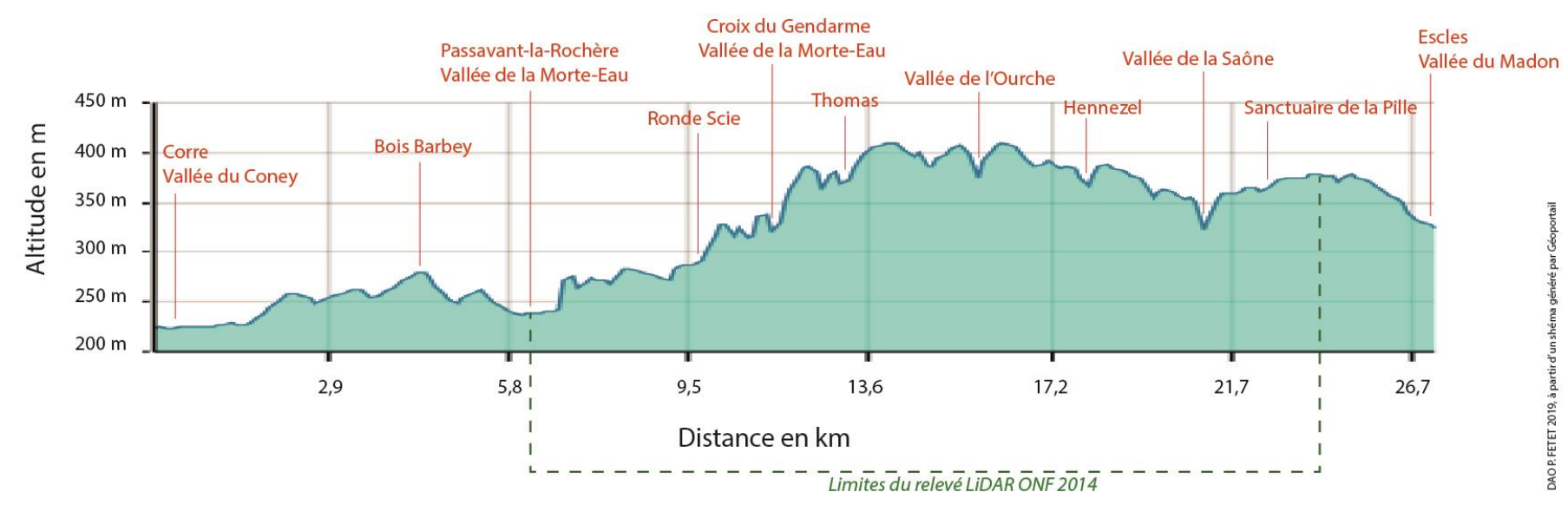

Fig. 4. Profil altimétrique de l'itinéraire Corre-Escles (DAO P. Fetet)

- Tronçon de bande de terre $\mathrm{n}^{\mathrm{o}} 1$

À Passavant-la-Rochère, la première trace se situe dans le Bois de la Côte, entre l'Étang Neuf et le chemin forestier du Morillon. Visible sur $500 \mathrm{~m}$, le relief rectiligne est ténu mais semble correspondre au fossé oriental de la bande de terre. Il a un azimut de $30^{\circ} \mathrm{E}$. Au sud du tronçon, le fossé amorce une courbure pour éviter une dépression due à un méandre de la Morte-Eau mais, passant au même endroit, le chemin rural de la Côte à la Rochère gêne la lisibilité.

- Tronçon de bande de terre ${ }^{\circ} 2$ (TB2, Fig. 5)

Dans le Bois de Passavant, les deux fossés-limites de la bande sont visibles de la Ronde Scie à la Croix du Gendarme sur une longueur de $800 \mathrm{~m}$. À la Ronde Scie, les fossés, visibles dès le bas de la pente, amorcent une première montée sur le plateau de la Vôge. À cet endroit, l'érosion due au passage des hommes et des animaux a entraîné la formation d'une dépression allongée d'environ $200 \mathrm{~m}$ comprise entre les deux bordures. On retrouve d'autres dépressions linéaires en forme de vagues, toujours entre les fossés, à la traversée d'un petit vallon formé par un affluent de la Morte-Eau. Puis les deux fossés s'arrêtent net sur le plateau, quelques mètres avant la rupture de pente de la vallée de ce ruisseau. Pour la totalité de ce tronçon, les fossés parallèles ont un azimut de $30^{\circ} \mathrm{E}$. 


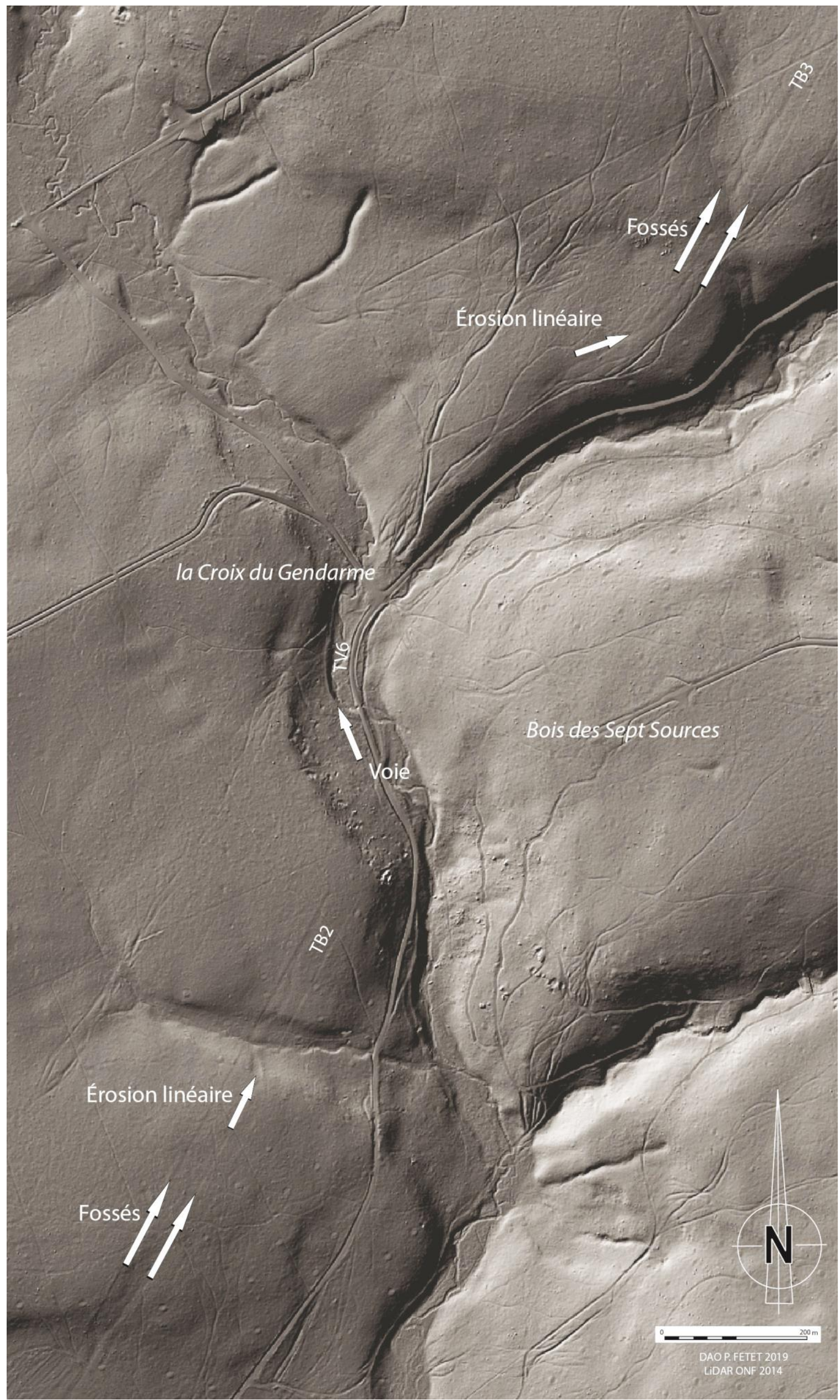

Fig. 5. Tronçons de bande de terre $n^{\circ} 2$ et 3 (TB2 et TB3) et tronçon de voie $n^{\circ} 6$ (TV6) à la Croix du Gendarme (LiDAR ONF 2014 et DAO P. Fetet) 


\section{- Évitement du ruisseau de Morte-Eau (fig. 5)}

Le tracé directeur rencontre trois fois le ruisseau de Morte-Eau : dans le village de Passavant, à la Rochère et au lieu-dit la Croix du Gendarme. Le LiDAR ne nous renseigne pas sur les deux premières traversées car ces zones ont été défrichées. Pour la troisième, à la fin du tronçon $\mathrm{n}^{\mathrm{o}} 2$, les fossés s'arrêtent à l'aplomb d'un dénivelé de $25 \mathrm{~m}$, point à partir duquel la bande de terre aurait dû traverser le cours d'eau. Les fossés étant inexistants sur l'autre rive, on peut faire l'hypothèse que le cheminement obliquait vers le nord pour descendre plus doucement en fond de vallée, traverser un ruisseau affluent et remonter avec une pente moyenne de $15 \%$ en restant sur la rive droite. Une rampe de quelques mètres de largeur sur la rive droite du ruisseau semble confirmer cette hypothèse ; de la sorte, l'itinéraire a pu éviter deux inconvénients au tracé théorique : une zone d'éboulis rocheux en fond de vallée et une montée suivie d'une descente immédiate sur le relief du Bois des Sept Sources.

- Tronçon de bande de terre $n^{0} 3$ (TB3, fig. 5)

Après le passage délicat du ruisseau de Morte-Eau, les deux fossés sont à nouveau visibles sur une longueur de $500 \mathrm{~m}$ avec un azimut de $31^{\circ} \mathrm{E}$. Du bas de la Croix du Gendarme, puis entre ces bordures rectilignes, on retrouve un cheminement sous forme d'une dépression linéaire quasiment dans l'axe central de la bande de terre. À l'approche d'un nouveau dénivelé important de $30 \mathrm{~m}$, les fossés disparaissent.
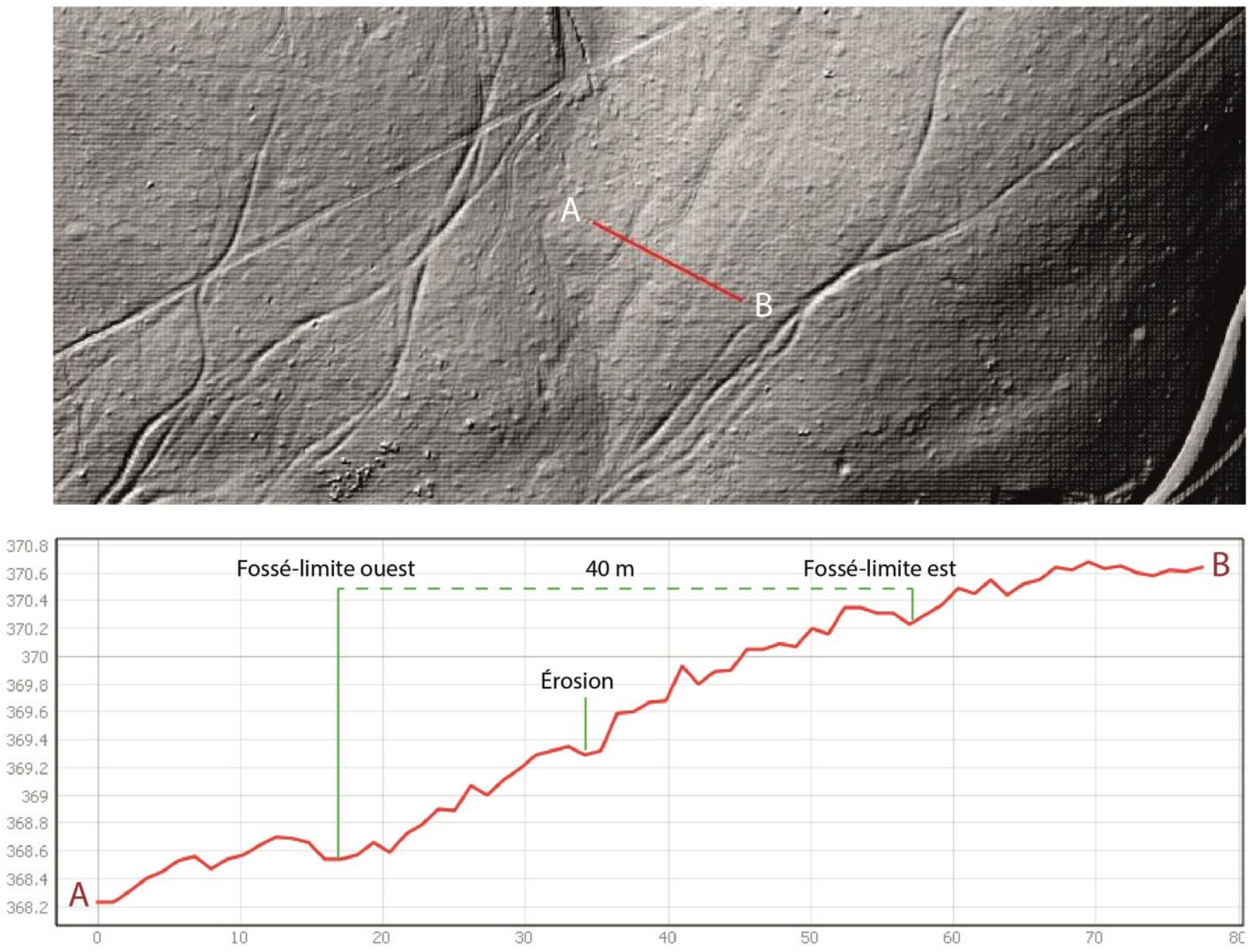

Fig. 6. Profil de la bande de terre $n^{\circ} 3$ (TB3) au nord-est de la Croix du Gendarme (Passavant-la-Rochère) 
À l'extrémité nord du tronçon $\mathrm{n}^{\mathrm{o}} 3$, une large dépression qui ne semble pas naturelle prend la direction de la source Saint-Walbert qui se trouve à $200 \mathrm{~m}$ à l'est de l'axe directeur. Il est possible que le cheminement évite ainsi le relief du Champ de l'Ermitage et reprenne ensuite l'axe directeur plus au nord au niveau du lieu-dit Thomas. Cependant, le défrichement de ce lieu habité ayant effacé toute trace de fossé, il est difficile de se prononcer définitivement ${ }^{7}$.

- Tronçon de bande de terre $\mathrm{n}^{\mathrm{o}} 4$ (TB4, fig. 6)

Au nord de Thomas, dans un premier temps, on ne distingue que le fossé oriental de la bande sur environ $200 \mathrm{~m}$. Quelques dizaines de mètres avant de traverser la tranchée ${ }^{8}$ de la Fontaine aux Cerfs, le fossé ouest réapparaît. Les deux bordures sont ensuite plus ou moins discernables jusqu'à la clairière de la Frison, dessinant un tronçon rectiligne de plus d'un kilomètre avec un azimut de $30^{\circ} \mathrm{E}$. Puis, à partir de la clairière, les fossés-limites deviennent invisibles.

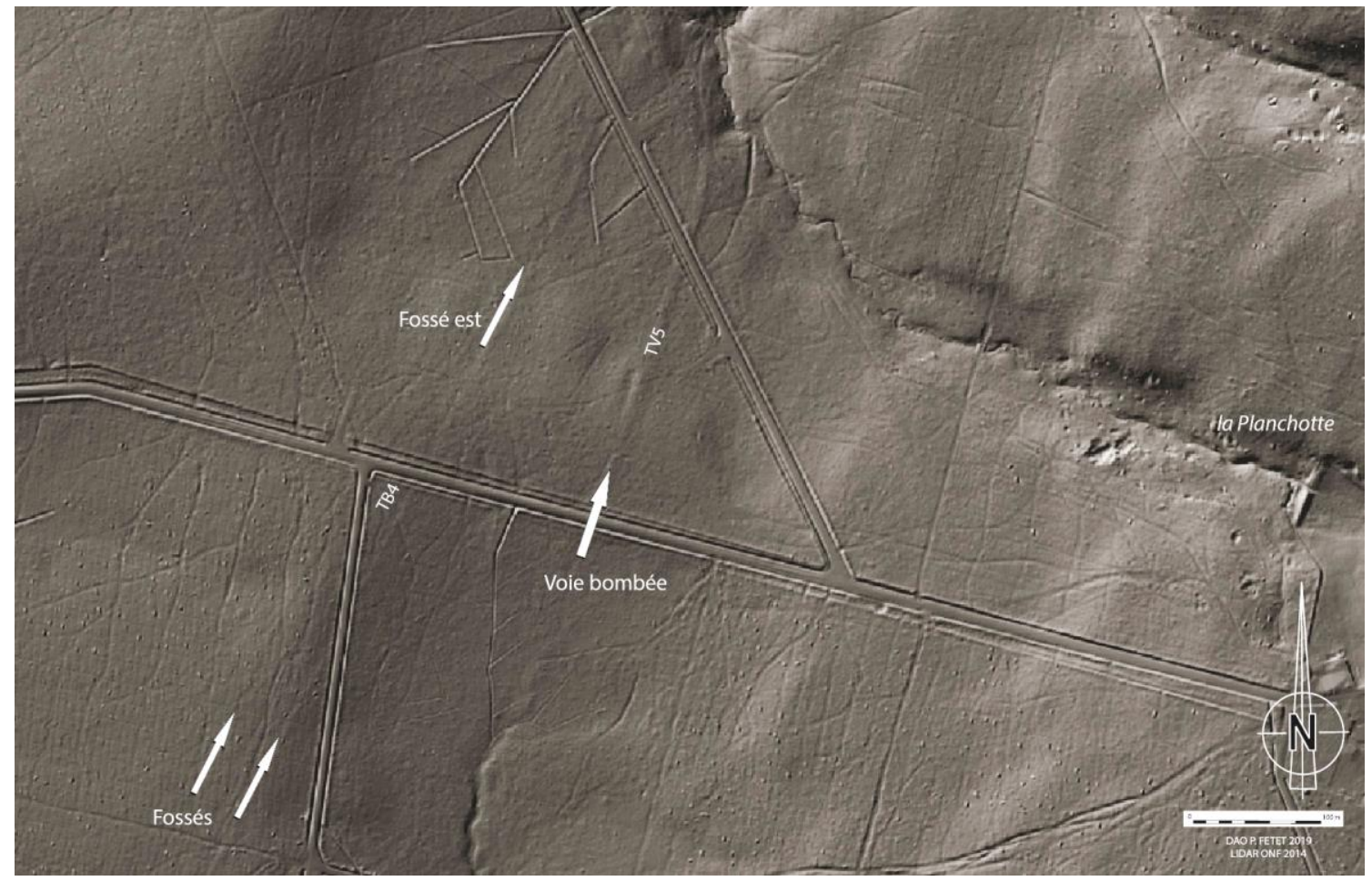

Fig. 7. Tronçon de bande de terre $n^{\circ} 4$ (TB4) et tronçon de voie $n^{\circ} 5$ (TV5) au nord de Thomas (LiDAR ONF 2014 et DAO P. Fetet)

\section{- Traversée de l'Ourche}

Le LiDAR ne donne pas d'information sur la manière dont la bande rectiligne approche la rive gauche de la vallée de l'Ourche qui a été défrichée. En revanche, on retrouve ses fossés sur la rive droite après un dénivelé de $25 \mathrm{~m}$ et une pente moyenne de $16 \%$. On ne distingue pas de rampe aménagée mais des marques d'érosion suggèrent un tracé en $\mathrm{S}$ pour traverser la vallée.

- Tronçon de bande de terre $\mathrm{n}^{\circ} 5$ (TB5, fig. 7)

\footnotetext{
${ }^{7}$ Il est à noter qu'au sud du Champ de l'Ermitage se trouvait autrefois une chapelle dédiée à saint Valbert (orthographié Walbert sur la carte IGN, mais il existe aussi les formes Waldebert, Waubert, Wambert, Vaubert ou Gaubert) dont l'origine remonte au Moyen Âge. Son emplacement est à quelques mètres d'une borne frontière non datée séparant la Franche-Comté de la Lorraine (Bertin et al. 2005).

${ }^{8}$ En Lorraine ou en Franche-Comté, la traversée d'une forêt conduisant à un défrichement par tronçons rectilignes a le nom de tranchée. Ce terme n'a donc rien à voir avec une excavation.
} 
Sur la rive droite de l'Ourche, au canton ${ }^{9}$ de Sainte-Marie, au niveau du musée de Clairey, les deux fossés sont à nouveau visibles sur une longueur de $700 \mathrm{~m}$ avec un azimut de $30^{\circ} \mathrm{E}$. Quasi parallèle à la bande de terre invisible sur le terrain, la tranchée Charlemagne - qui est la route départementale 5D menant d'Hennezel à Clairey - la recouvre au nord, ne laissant plus apparaître que le fossé ouest. La longueur totale du tronçon $\mathrm{n}^{0} 5$ est ainsi reconnaissable sur $1,3 \mathrm{~km}$ avant d'être effacée par le défrichement du village d'Hennezel à partir du croisement avec la route de Thiétry.

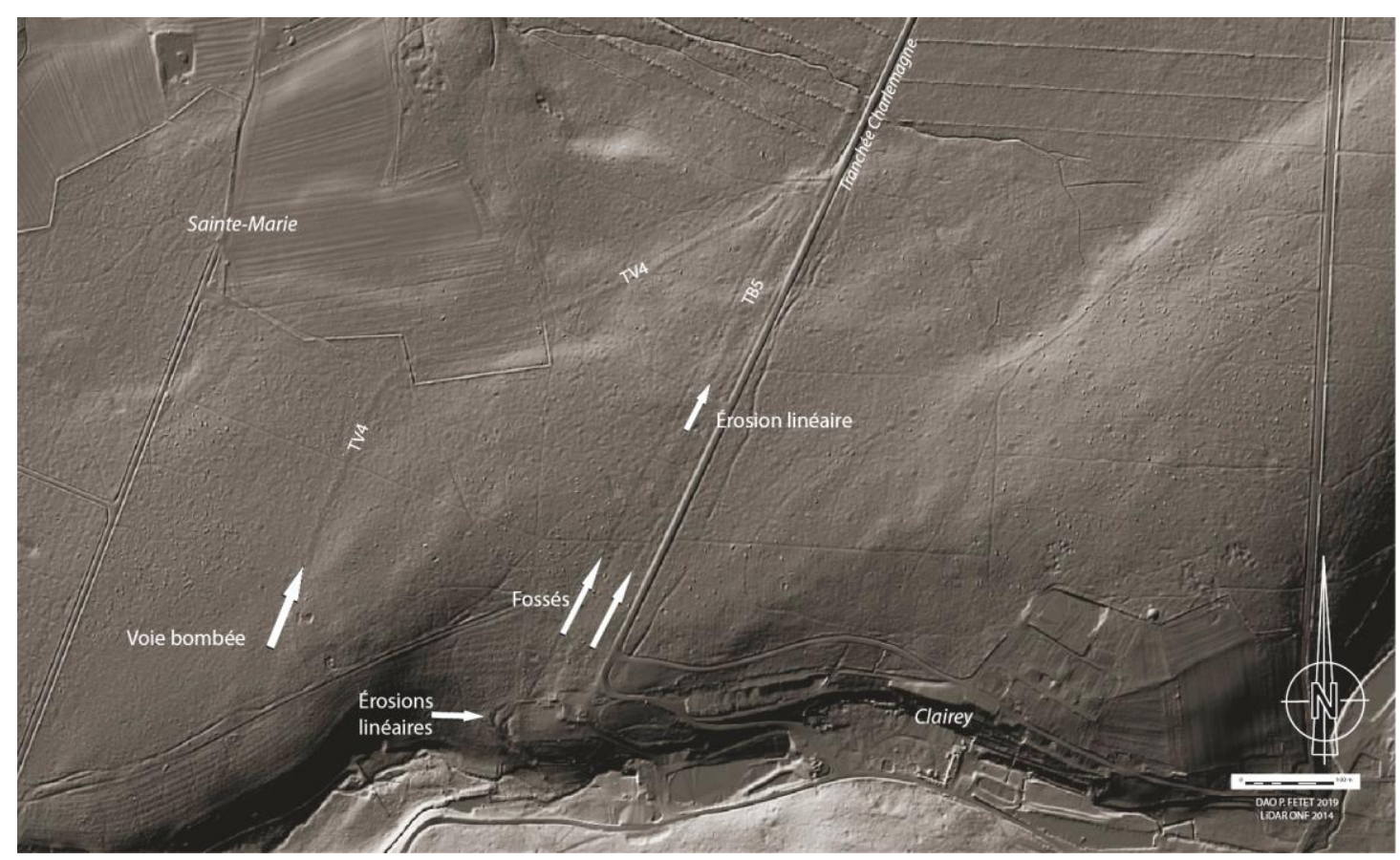

Fig. 8. Tronçon de bande de terre $n^{\circ} 5$ (TB5) et tronçon de voie $n^{\circ} 4$ (TV4) au canton de Sainte-Marie (LiDAR ONF 2014 et DAO P. Fetet)
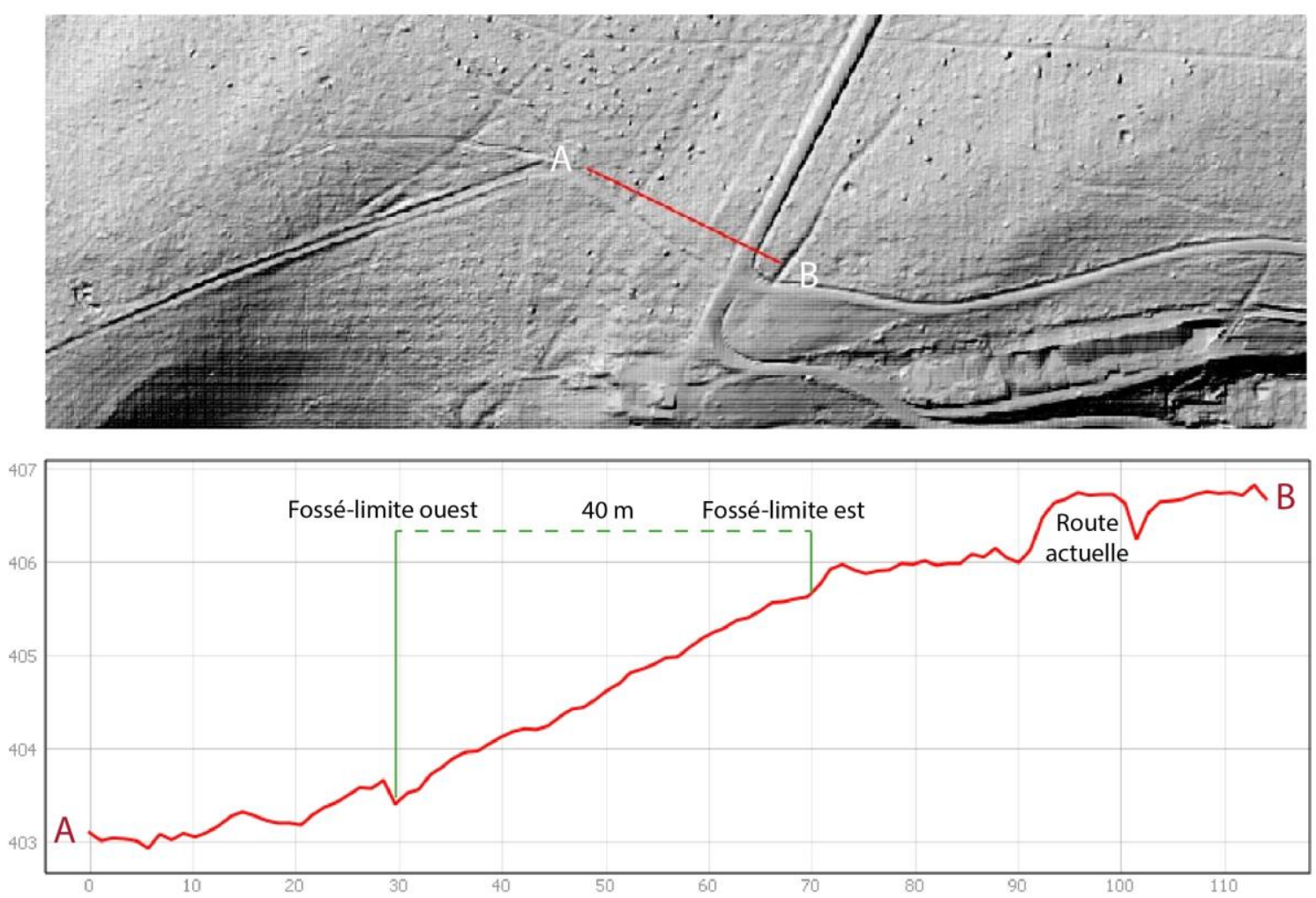

Fig. 9. Profil de la bande de terre $n^{\circ} 5$ (TB5) au niveau du musée de Clairey (Hennezel)

\footnotetext{
${ }^{9}$ Ce terme « canton » était utilisé autrefois pour désigner la subdivision territoriale d'une forêt.
} 
- Tronçon de bande de terre $\mathrm{n}^{\circ} 6$ (TB6, fig. 10 et 11)

Après $1,2 \mathrm{~km}$, on retrouve les fossés au nord des Champs de la Chapelle dans le même alignement que le tronçon $\mathrm{n}^{\circ} 5$. Les travaux forestiers consécutifs à la tempête de 1984 semblent avoir diminué la netteté des tracés mais ceux-ci restent visibles jusqu'à la clairière de Clairefontaine avant d'avoir traversé sans difficulté apparente le ruisseau qui provient de l'étang De Finance. Passée la petite zone de défrichement, les deux fossés se poursuivent jusqu'à la limite du plateau, coupé par la vallée de la Saône. Nous avons choisi de ne faire qu'un seul tronçon pour ces trois fragments car ni la coupure du petit ruisseau ni celle de la clairière n'ont affecté l'orientation de la bande de terre. La longueur totale du tronçon $\mathrm{n}^{\circ} 6$ est de $2,4 \mathrm{~km}$ avec un azimut de $29^{\circ} \mathrm{E}$.

\section{- Traversée de la Saône}

La traversée de la vallée de la Saône est sans doute le plus grand obstacle du parcours car elle comporte un dénivelé de $32 \mathrm{~m}$ avec une pente moyenne de $20 \%$ pour la rive gauche et de près de $30 \%$ pour la rive droite. Le cheminement de la rive gauche emprunte un couloir en pente modérée qui a subi une large érosion dans sa partie supérieure. La pente vers la Saône semble naturelle, excepté le fait qu'on y a probablement supprimé quelques enrochements. En revanche, la rive droite aurait été inaccessible sans la création d'une rampe importante repérée grâce au LiDAR. On distingue très bien son terrassement sur une centaine de mètres dans sa partie supérieure avec une largeur d'environ $16 \mathrm{~m}$ et une pente moyenne de $14 \%$. Cependant, la partie inférieure reste douteuse car elle a été modifiée par un chemin transverse. Au sommet de la rampe, la zone de la Bataille est défrichée.

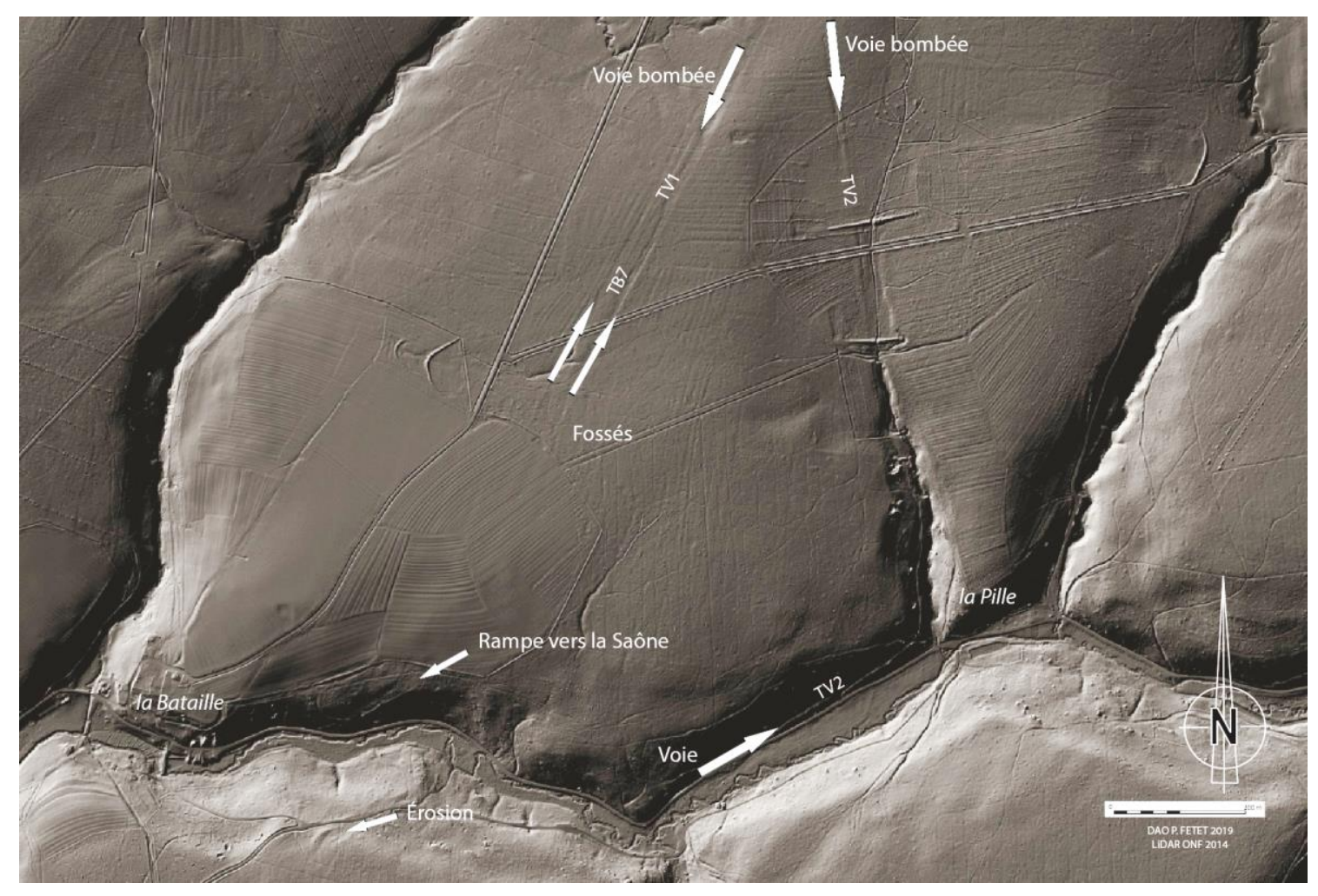

Fig. 10. Tronçon de bande de terre $n^{\circ} 7$ (TB7) et tronçons de voie $n^{\circ} 1$ et 2 (TV1 et TV2) dans la forêt de la Pille (LiDAR ONF 2014 et DAO P. Fetet) 

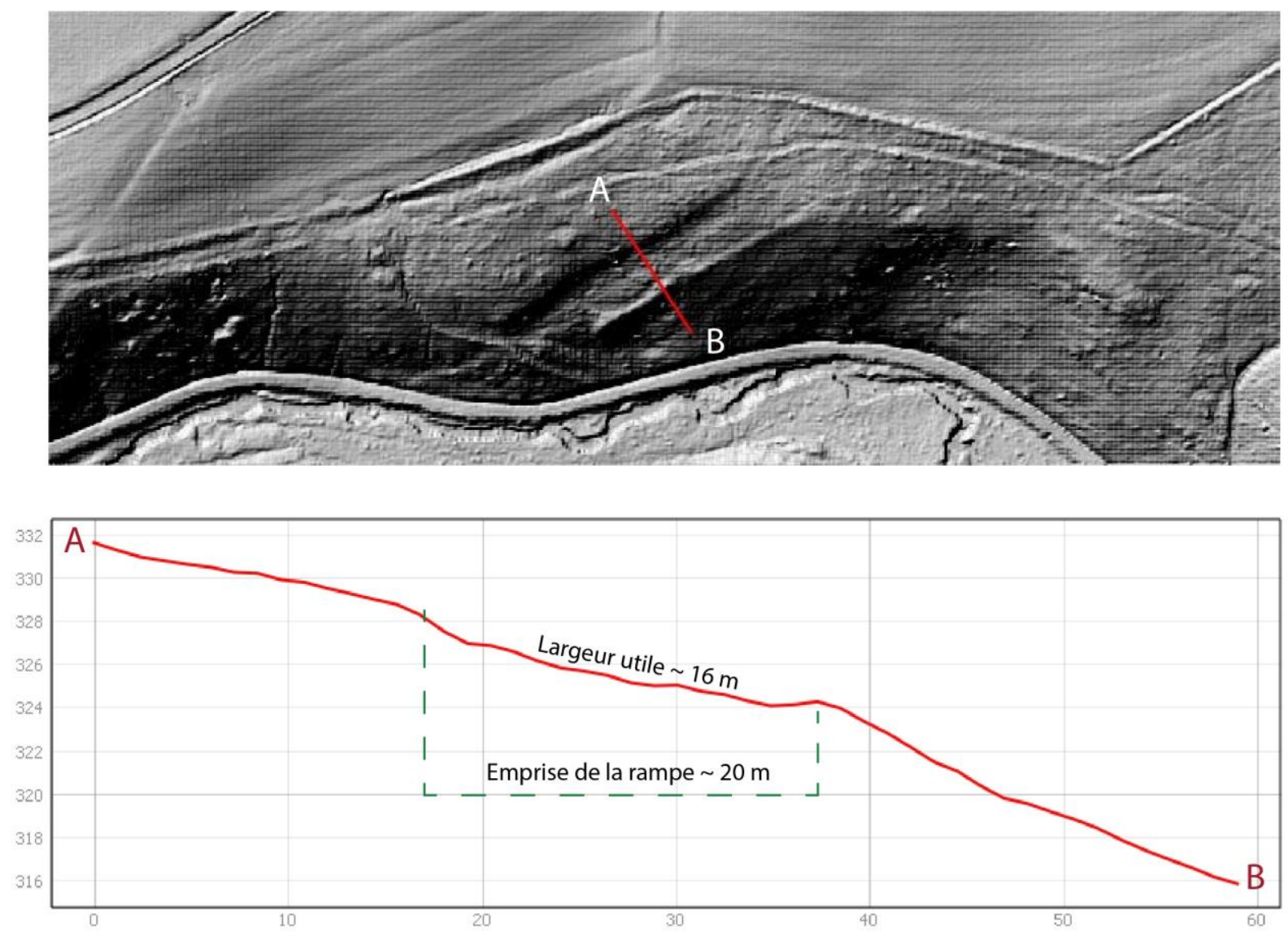

Fig. 11. Profil de la rampe de la rive droite de la Saône à la Bataille (Vioménil)

\section{- Tronçon de bande de terre $\mathrm{n}^{\mathrm{O}} 7$ (TB7, fig. 8 et 9)}

À la sortie de la clairière de la Bataille, les deux fossés réapparaissent à partir de la tranchée de la Pille et marquent un nouveau tronçon rectiligne de $2,4 \mathrm{~km}$ avec un azimut de $30^{\circ} \mathrm{E}$. Les fossés sont toutefois différents de ceux perçus de Passavant-la-Rochère à la haute vallée de la Saône. Au lieu de former un sillon laissant une empreinte d'environ 5 à $6 \mathrm{~m}$ de largeur, les fossés de la bande de terre sont confondus avec ceux d'une voie centrale (TV1). Chacun d'eux a une emprise de 14 à $15 \mathrm{~m}$ et le relief sur lequel est construite la voie a une emprise d'environ $11 \mathrm{~m}$. Ainsi, l'orientation et la largeur de la bande de terre sont les mêmes qu'au sud mais, à l'approche de l'agglomération d'Escles qui se trouve à $9 \mathrm{~km}$ de la Saône, le traitement est différent : les fossés sont larges et une voie centrale est aménagée ${ }^{10}$.

Ce tronçon est également remarquable par la présence d'un sanctuaire dédié à Mercure en bordure de voie. Il est situé contre la bordure ouest de la bande et marque le carrefour de plusieurs voies : celle qui vient d'être décrite, au centre de la bande rectiligne (TV1), celle d'une voie se raccordant à la première qui sera décrite plus bas (TV2) et celle d'Attigny à Vioménil (Fetet \& Gaffiot 2016).

- Caractéristiques globales de la bande de terre

Après avoir décrit précisément cet espace révélé par le LiDAR, nous pouvons résumer ici ses principales caractéristiques. Tout d'abord, cet axe conserve une même direction d'azimut $30^{\circ} \mathrm{E}$ à 1 degré près sur tout le parcours observé et même au-delà puisqu'il est la continuité d'axes routiers antiques reconnus à Corre et à Escles. Toutefois, au sud de la haute vallée de la Saône, cette bande semble n'avoir été matérialisée que par ses fossés-limites, séparés l'un de l'autre de $40 \mathrm{~m}$ environ,

\footnotetext{
${ }^{10}$ La voie transjurassienne repérée au nord de Pontarlier sur le plateau de la Vrine a les mêmes caractéristiques : ses fossés latéraux s'étendent jusqu'à environ 20 mètres de l'axe du remblai central, matérialisant le domaine public et reflétant l'importance de cet axe dans le réseau routier (Bichet et al. $2019: 16)$. 
puisqu'aucune trace de chaussée n'y a été repérée pour l'instant, excepté la voie décrite plus bas qui la croise régulièrement. La longueur totale de visualisation de cette bande est de $8,5 \mathrm{~km}$, ce qui représente la moitié de la longueur analysée qui s'élève à $17,8 \mathrm{~km}$. Sur ces $8,5 \mathrm{~km}$, aucun fossé-limite n'a pour l'instant été repéré sur le terrain car, d'une part, ces reliefs ne sont pas perceptibles à l'œil et, d'autre part, la végétation forestière empêche toute vision avec du recul. Ce n'est que lorsque la bande de terre rencontre une pente que des traces matérielles supplémentaires apparaissent : en cas de déclivité, une érosion linéaire en creux est souvent visible entre les fossés, ce qui révèle la fréquentation de cet espace sous la forme d'un chemin. Ces traces, elles aussi ténues et non encore explorées sur le terrain, sont visibles par endroits grâce au LiDAR sur un total cumulé d'environ $1,2 \mathrm{~km}$. Elles ne représentent que $6,7 \%$ de la longueur analysée. Une trace matérielle plus concrète apparaît quand les fossés rencontrent des vallées : la rampe. Repérable sur le terrain, elle peut prendre deux aspects différents. Celle de la descente vers la Saône est massive et rectiligne (environ $16 \mathrm{~m}$ de largeur sur $100 \mathrm{~m}$ de longueur), alors que les autres, d'une largeur inférieure, ont plutôt une forme courbe présentant un creux marqué.

Par ailleurs, la forêt et l'occupation du sol sont utiles pour proposer une datation de ces aménagements. En effet, la couverture forestière a joué un rôle protecteur qui a permis la préservation de traces imperceptibles sur le terrain. Suite à l'abandon de ces fossés et de ce chemin, la forêt s'est installée durablement jusqu'à aujourd'hui car sur l'ensemble de l'emprise de la bande de terre ainsi définie, seuls les défrichements ont gommé complètement ces traces. Or ces défrichements ont commencé suite à l'installation de verreries attestées au moins depuis le XIV siècle (Ladaique 2009). La création de ces fossés n'a donc pu être réalisée qu'avant la fin du Moyen Âge. Par ailleurs, l'absence totale de reprise de cet axe dans le cadastre ou de persistance dans la voirie moderne nous conduit à considérer ce couloir comme extrêmement ancien. En outre, l'ensemble de ces constatations pour ces $17 \mathrm{~km}$ et le fait que l'axe directeur relie deux agglomérations gallo-romaines et s'aligne avec une voie pavée rectiligne, invisible dans le paysage mais reconnue archéologiquement de l'époque gallo-romaine en plusieurs endroits sur le territoire d'Escles, nous font considérer cette bande comme datant de l'Antiquité.

Enfin, ce couloir large de $40 \mathrm{~m}$ évoque un espace public où il serait interdit de cultiver, comme le suggère Gérard Coulon qui, pour les voies, fait le parallèle avec la législation de la protection des aqueducs que l'on connait, entre autres, par cette inscription de Chagnon (Loire) : «Par ordre de l'empereur César Trajan Hadrien Auguste, personne n'a le droit de labourer, de semer ou de planter dans cet espace de champ qui est destiné à la protection de l'aqueduc.» (Coulon 2007: 86) La différence est qu'au sud de la haute vallée de la Saône, on ne distingue pas de voie terrestre centrale, ni en prospection pédestre, ni avec le LiDAR, hormis ces érosions linéaires que l'on ne visualise que dans les pentes et qui dévoilent un cheminement. Cette observation nous conduit à penser que les fossés considérés ici délimitent un espace sans aménagement routier particulier. $\mathrm{Si}$ cet espace était entièrement défriché, ce dont on ne peut, pour l'heure, être certain, sa largeur importante pouvait être utile pour le déplacement de troupeaux qui, en même temps que de se déplacer, pouvaient entretenir cet espace ouvert par le pâturage. Pour ce qui concerne le déplacement des armées, puisque cet axe SaôneMoselle était important pour rejoindre le limes (Feugère \& Bonnamour 1996: 145), cette largeur pouvait également servir à limiter l'efficacité d'éventuelles embuscades.

Au nord de la haute vallée de la Saône, nous l'avons développé plus haut dans la description du tronçon de bande de terre $\mathrm{n}^{\circ} 7$, les fossés sont plus larges et une chaussée pavée occupe la partie centrale. Ce traitement différent de ce même axe correspond à un changement d'occupation du sol marqué par une anthropisation antique accrue avec la présence du sanctuaire de La Pille, la trace de parcellaires de cultures et la proximité de la localité d'Escles. Ce changement entre ce qui pourrait être un milieu forestier et un espace défriché, qu'on observe à partir de la traversée de la haute vallée de la Saône, révèle peut-être aussi la proximité de la limite administrative entre les cités leuque et séquane. 
La plupart des tronçons de voies étaient connus grâce aux prospections pédestres. Cependant, le LiDAR apporte des précisions sur leur tracé et révèle de nouveaux segments. Parmi tous ces tronçons, seul le premier (TV1) est attesté de l'Antiquité car il est archéologiquement relié au sanctuaire de la Pille. Pour les autres tronçons, cette datation antique, même si elle est probable en raison de l'observation de la structure de la voie, n'a pas pu encore être prouvée.

- Tronçon de voie $\mathrm{n}^{\mathrm{o}} 1$ (TV1, fig. 8 et 9 )

Observons les tronçons de voies d'Escles à Corre. Le premier tronçon connu est celui qui va du village d'Escles à la forêt de la Pille. Il est totalement rectiligne sur une longueur de $5 \mathrm{~km}$ et sa partie visible sur le LiDAR montre une chaussée en relief bombé centré sur l'axe de la bande de terre allant au-delà du sanctuaire de la Pille. Si les fossés borduriers sont bien visibles jusqu'à la lisière de la forêt, la voie quant à elle semble avoir été démantelée jusqu'à une centaine de mètres au sud du sanctuaire. On peut donc supposer qu'elle allait à l'origine jusqu'à la rupture de pente du vallon encaissé de la Saône et se raccordait à la rampe descendant vers la rivière. Sur la totalité de la bande rectiligne entre Passavant et Vioménil, c'est le seul tronçon de voie attesté sur le terrain que nous ayons décelé centré entre les fossés. Au sud de la vallée de la Saône, les tronçons de voies observés ne semblent plus être en lien avec la bande de terre, excepté peut-être au canton de Sainte-Marie.

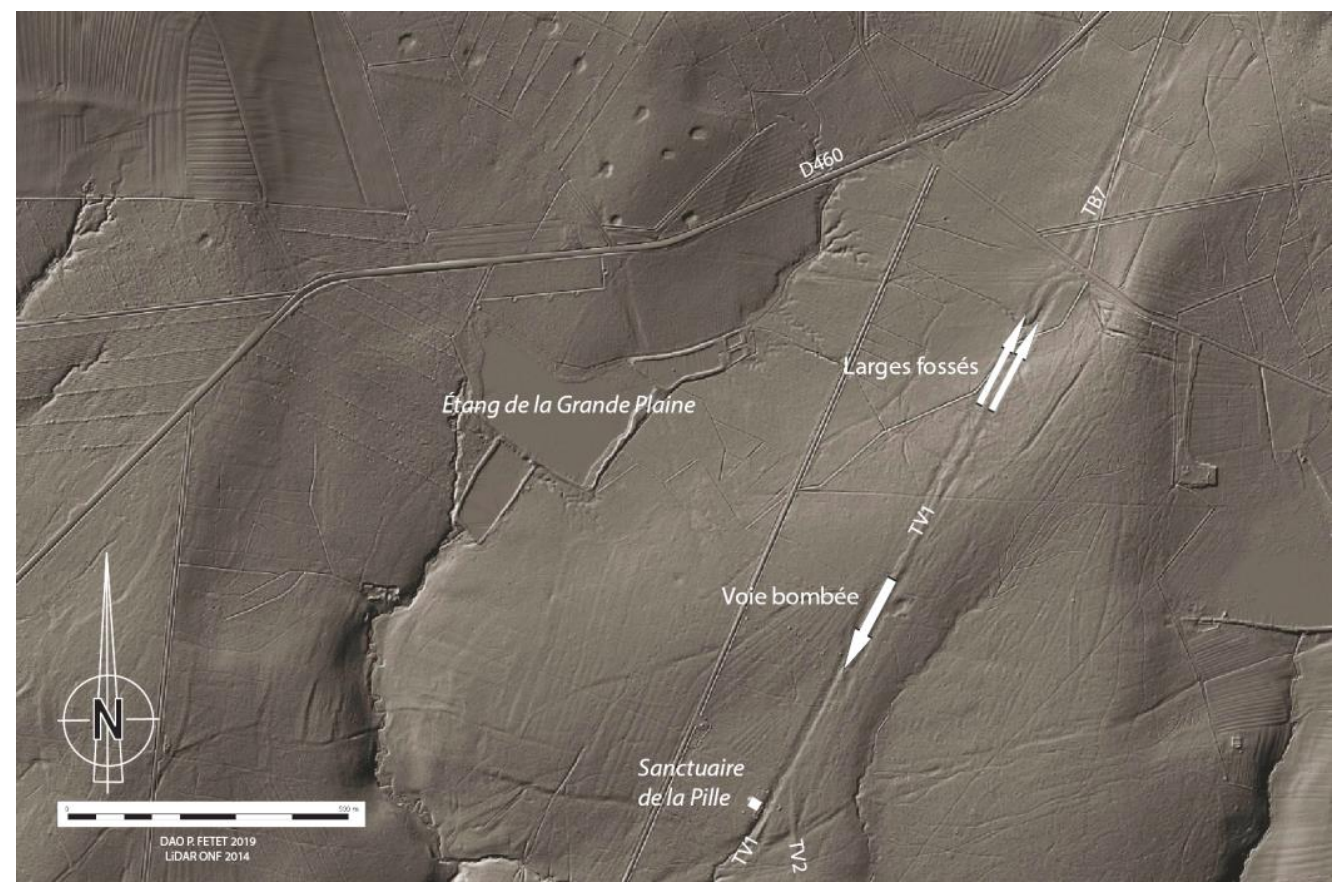

Fig. 12. Tronçons de voies $n^{\circ} 1$ et 2 (TV1 et TV2) et tronçon de bande de terre $n^{\circ} 7$ (TB7) près du sanctuaire de la Pille (LiDAR ONF 2014 et DAO P. Fetet)

- Tronçon de voie $\mathrm{n}^{\mathrm{o}} 2$ (TV2, fig. 8,9 et 10$)$

Le deuxième tronçon de voie est d'une autre nature. Il débute au carrefour situé près du sanctuaire de La Pille en se déviant de l'axe directeur pour emprunter un vallon en pente douce. C'est donc une voie secondaire par rapport à la voie principale d'Escles. La coupe de cette voie montre une structure légèrement différente de celle du premier tronçon rectiligne : il n'apparaît qu'une seule couche de construction, constituée de blocs de grès peu agencés sur une largeur d'environ $6 \mathrm{~m}$ (Bertin et al. 1997). Toutefois, il reste possible que les blocs de grès de cette voie aient été récupérés à cet endroit pour construire des chaussées d'étangs. Cette déviation permet de traverser la vallée de la Saône en évitant les forts dénivelés. La voie descend en pente douce le vallon des trois anciens étangs de la Pille sous lesquels elle disparaît. Puis, grâce à des observations de terrain décrites plus haut, on sait qu'à la rencontre de la vallée de la Saône, elle tourne vers l'ouest pour descendre en rampe le versant abrupt 
de la rive droite. Elle traverse ensuite le cours d'eau en biais et remonte la rive gauche pour gagner le haut du plateau. On comprend bien que le but de cette voie non rectiligne est d'offrir une pente maîtrisée accessible aux véhicules. Une fois la vallée traversée, la voie prend la direction du sud-ouest, sans pour autant se confondre avec la bande de terre qui lui est parallèle. On la perd à la limite du défrichement du hameau de Clairefontaine.

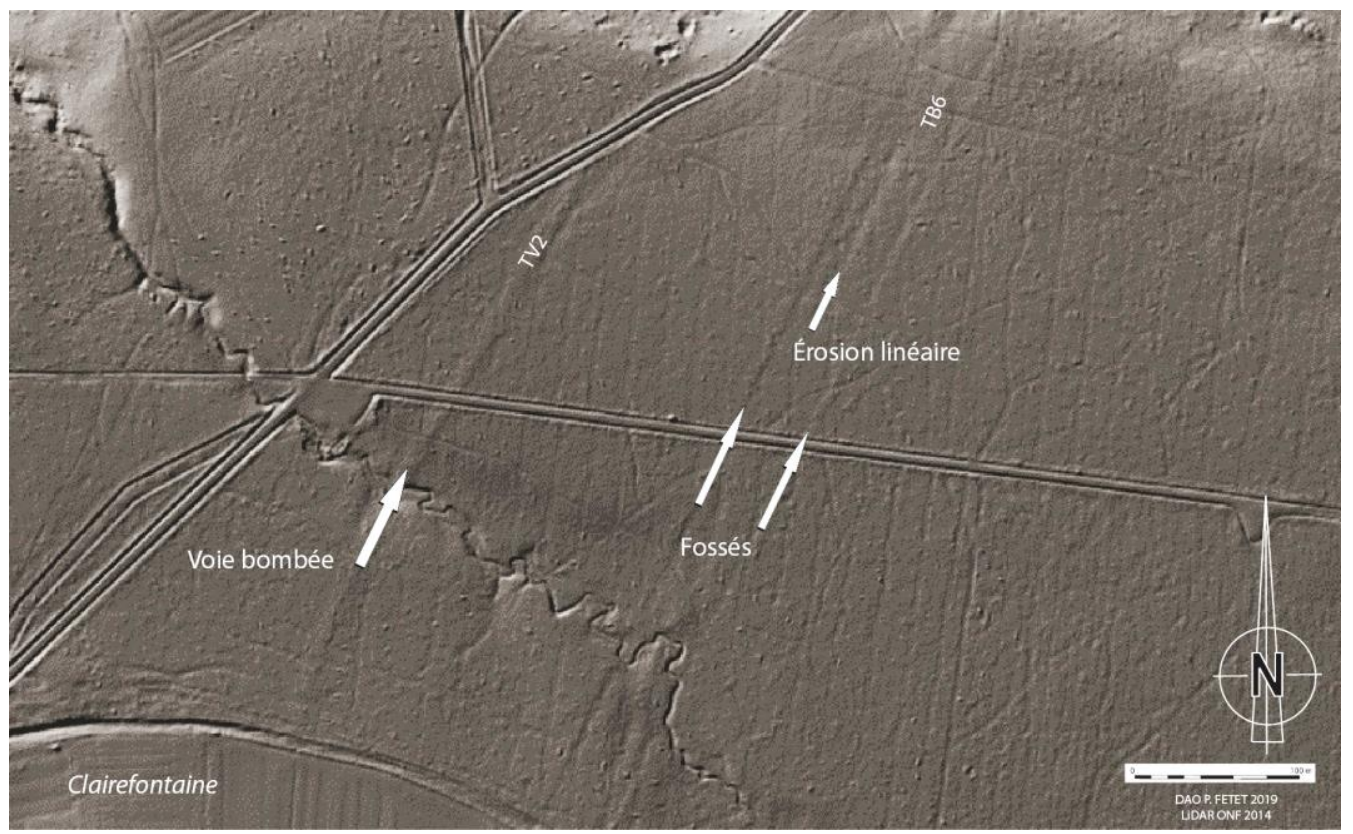

Fig. 13. Tronçon de voie $n^{\circ} 2$ (TV2) et tronçon de bande de terre $n^{\circ} 6$ (TB6) dans le bois de Clairefontaine (LiDAR ONF 2014 et DAO P. Fetet)

- Tronçon de voie $\mathrm{n}^{\mathrm{o}} 3$ (TV3, fig. 11)

La voie secondaire réapparaît à l'approche d'Hennezel au niveau du carrefour de la route forestière de Clairefontaine et du chemin de la chapelle Saint-Joseph. On distingue sa forme linéaire bombée sur environ $800 \mathrm{~m}$. Elle rejoint la bande de terre comme pour s'y raccorder mais, arrivée presque en position centrale par rapport aux deux fossés-limites, elle amorce un virage vers le sud, recouvre le fossé oriental et disparaît au niveau du défrichement.

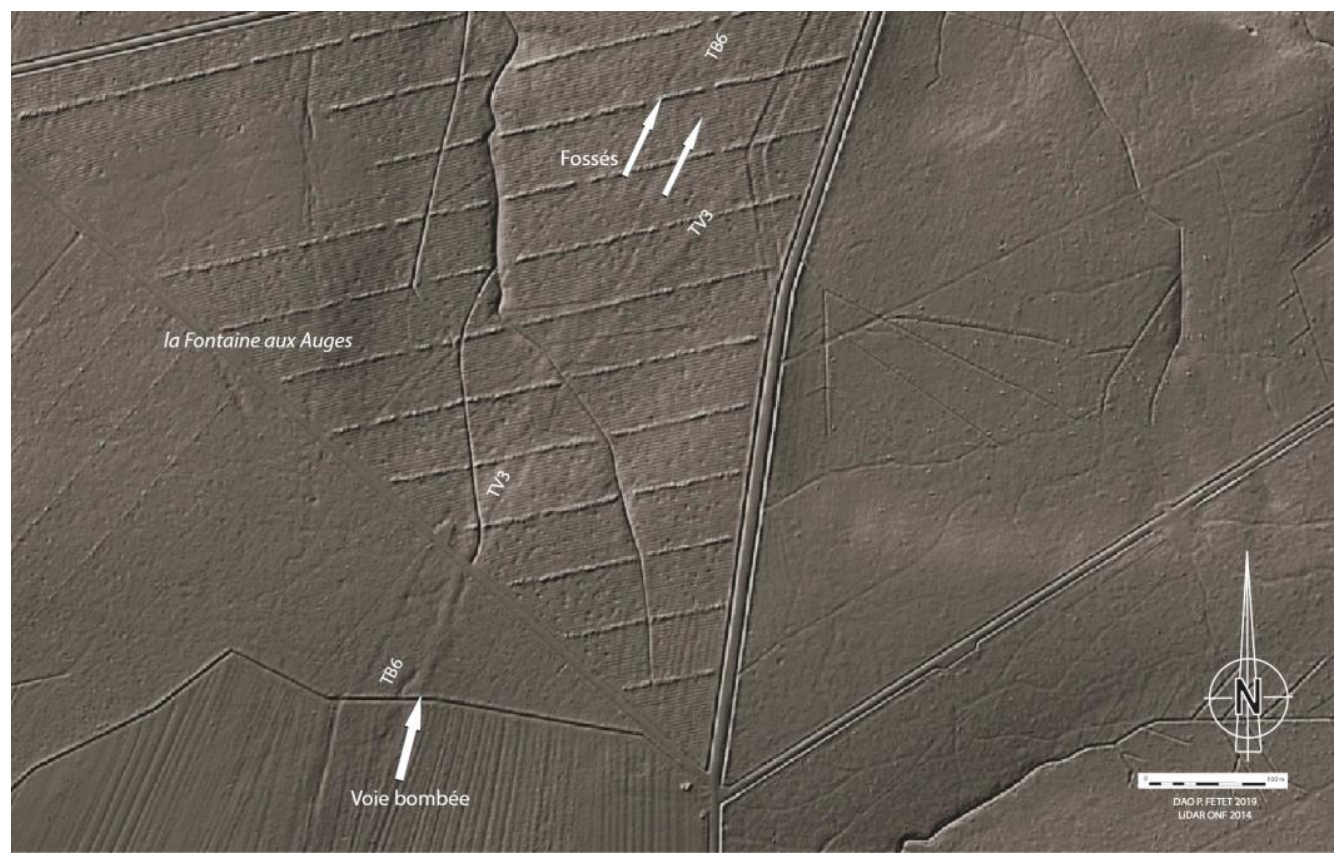

Fig. 14. Tronçon de voie $n^{\circ} 3$ (TV3) et tronçon de bande de terre $n^{\circ} 6$ (TB6) à la Fontaine aux Auges (LiDAR ONF 2014 et DAO P. Fetet) 
- Tronçon de voie $\mathrm{n}^{\mathrm{o}} 4$ (TV4, fig. 7)

On retrouve le relief de la voie au niveau du canton de Sainte-Marie. Elle semble avoir pris une position centrale par rapport aux fossés de la bande rectiligne sur quelques dizaines de mètres mais ce relief ténu est trop près de la route actuelle pour pouvoir l'attester. Elle s'écarte de la tranchée Charlemagne en se dirigeant vers l'ouest sur $500 \mathrm{~m}$ puis bifurque vers le sud et disparaît au bout de $200 \mathrm{~m}$. On ne sait donc pas où ni de quelle manière elle traverse la vallée de l'Ourche.

- Tronçon de voie $\mathrm{n}^{\mathrm{O}} 5$ (TV5, fig. 6)

La voie est à nouveau visible au sud de la clairière de la Frison, sortant du vallon du ruisseau provenant de la Planchotte. On perd quasiment sa trace au niveau de la route forestière reliant la Planchotte et la Sybille. Puis, dans le prolongement de sa direction vers le sud, on remarque encore un bombement d'une centaine de mètres de longueur au niveau du ruisseau de la fontaine aux Cerfs. Les constructeurs semblent donc avoir pris le parti de suivre le fond du vallon qui débouche sur le hameau Thomas. À ce niveau, la voie se trouve à $200 \mathrm{~m}$ environ à l'est de la bande de terre.

- Tracé de voie n ${ }^{\circ} 6$ (TV6, fig. 5)

Un tronçon d'une centaine de mètres est visible à la Croix du Gendarme, amorçant un virage en fond de vallée sur la rive droite du ruisseau de Morte-Eau, en aval du confluent. Sur le terrain, on peut voir un agencement de blocs de grès servant de contrefort à la voie pour éviter qu'elle ne soit sapée par le cours d'eau. Jusqu'à la Rochère, on ne retrouve pas de trace de cette voie qui a pu être recouverte par la voirie actuelle ou effacée par l'activité humaine.

- Tracé de voie $\mathrm{n}^{\mathrm{0}} 7$

Le dernier tronçon visible se situe près de la maison forestière de la Perrière. Sur les $300 \mathrm{~m}$ où il est discernable, il est rectiligne et a un azimut d'environ $21^{\circ} \mathrm{E}$. On rencontre ce toponyme - Perrière deux autres fois sur le tracé de la voie de Corre à Escles : le Bois des Perrières à Vougécourt et les Perrières à Escles. Plus au nord, sur le tracé théorique de cette voie rejoignant la Moselle, on trouve également un Bois Perrière à Ville-sur-Illon. Ce toponyme semble donc avoir un lien avec la récupération du pavage de la voie romaine.

\section{- Caractéristiques globales des voies}

Avec le LiDAR, les traces de voies sont ainsi visibles sur une longueur de $6 \mathrm{~km}$, soit environ un tiers de la distance étudiée de 17,8 km. La visibilité est donc moindre que celle des fossés-limites de la bande de terre. Cela peut s'expliquer par le fait que cette voie a fait l'objet de récupérations de ses blocs de grès à de nombreux endroits, en particulier à proximité des clairières habitées dès la fin du Moyen Âge ou à l'Époque moderne.

Au sud de la haute vallée de la Saône, la voie est reconnaissable avec le LiDAR par un léger bombement, sans qu'apparaissent clairement des fossés bordiers. Son emprise est de 10 à $12 \mathrm{~m}$ et sa largeur de voie de roulement est d'environ $6 \mathrm{~m}$, telle qu'elle a été reconnue lors de son nettoyage à Hennezel ${ }^{11}$. Les nombreuses lacunes concernant son relevé ne permettent pas d'avoir une connaissance exhaustive de son tracé. De plus, une absence de données sur plusieurs kilomètres ne nous permet pas non plus d'en attester la continuité. Toutefois, la persistance de caractéristiques visuelles avec le LiDAR et sa position toujours proche de l'axe de la bande de terre nous font penser qu'elle est unique et que ses concepteurs ont eu le même objectif que ceux qui ont aménagé la bande de terre rectiligne : relier Corre à Escles.

\footnotetext{
${ }^{11}$ Initiées par Bernard Delémontey dès 1986, des opérations de décapages des blocs de grès ont eu lieu durant quelques années et ont permis de rendre visible cette voie sur une cinquantaine de mètres.
} 
Cela dit, rien ne prouve pour l'heure que cette voie terrestre soit de l'Antiquité. Seuls quelques indices le laissent entrevoir : son rattachement à la voie antique au niveau du carrefour du sanctuaire de la Pille et son orientation générale qui coïncide à la liaison des deux localités gallo-romaines. Un élément n'est toutefois pas favorable à cette hypothèse : la voie semble ignorer le tracé de la bande de terre rectiligne, sauf au nord, au-delà de la haute vallée de la Saône. Bien que l'Antiquité couvre plusieurs siècles, cette observation nous incite à rester prudents sur sa datation en conservant la possibilité d'une construction allant de l'Antiquité au Moyen Âge.

\section{Interprétation des données}

La comparaison entre les résultats des prospections pédestres durant les deux dernières décennies et les informations fournies par le LiDAR de 2014 montre que cette technologie ouvre la porte à une compréhension accrue de l'occupation du sol. Pour ce qui concerne le territoire entre Corre et Escles, elle permet de savoir que des arpenteurs ont réalisé des visées sur des dizaines de kilomètres tout en conservant la même direction de $30^{\circ} \mathrm{E}$ à 1 degré près. Cela signifie que cet itinéraire est le résultat d'une réflexion aboutie avec un objectif unique : relier deux points géographiques, en l'occurrence deux rivières, par un axe le plus court possible, et ce quel que soit le relief, ce qui répond tout à fait aux besoins d'un chemin de portage.

L'observation des tracés mis en évidence par le LiDAR permet de constater que deux cheminements étaient possibles pour passer de la Saône à la Moselle, sans que l'on puisse pour autant assurer leur contemporanéité : un portage pédestre ou animalier individuel et un portage routier. Le premier, en ligne droite sur un terrain probablement dégagé sur une quarantaine de mètres de largeur, était le plus court mais nécessitait d'emprunter des pentes raides à la traversée des vallées. Son utilisation a laissé de nombreuses traces d'érosion, bien visibles dans les dénivelés. Le second, utilisant des pentes douces, était un peu plus long mais permettait de circuler avec des charriots transportant des charges lourdes, comme cela est attesté par les ornières laissées dans le grès et retrouvées à plusieurs endroits du parcours. La largeur de sa chaussée, de 4 à $6 \mathrm{~m}$, permettait aisément à deux véhicules de se croiser. Bien que possiblement créés à deux époques différentes, les deux parcours de ce même itinéraire se rejoignaient dans la forêt de la Pille au niveau du sanctuaire de Mercure. De là, la voie rectiligne unique aboutissait à l'agglomération d'Escles qui se trouve au milieu de l'axe Corre-Portieux.

Celui-ci, dans l'hypothèse où il a existé intégralement, aura nécessité une planification de travaux sur $54 \mathrm{~km}$ que seule une puissance publique ou une riche corporation a pu financer. Aux points supposés de transbordement, Corre et Portieux, les cours d'eau bénéficient d'un plus fort débit avec des affluents : le Coney pour la Saône et le Durbion pour la Moselle. En créant cet axe, le choix aura donc pu être de privilégier non pas le plus court chemin entre les deux rivières mais le plus court chemin permettant de relier deux ports pouvant accueillir des bateaux d'une certaine capacité, donc nécessitant un certain niveau d'eau.

\section{Questions et perspectives}

On peut se poser la question de la contemporanéité des deux parcours. La voie n'étant pas systématiquement centrée sur la bande de terre rectiligne, il est possible que les aménagements aient été effectués en plusieurs phases. La voie pourrait être postérieure à la bande de terre car sa structure semble combler par deux fois le fossé-limite oriental de la bande de terre dans le secteur de la Fontaine aux Auges à Hennezel (fig. 11). Il est à noter aussi qu'un défrichement de $40 \mathrm{~m}$ de large sur une vingtaine de kilomètres a pu être difficile à entretenir et que la forêt a pu assez rapidement regagner le terrain.

Par ailleurs, le LiDAR de 2014 ne couvre pas une zone assez étendue pour nous renseigner sur les aménagements routiers à proximité de l'agglomération de Corre ni sur son raccordement avec la voie 
antique de Martinvelle à Lignéville ${ }^{12}$ (Fetet 2016). La présence ou non des fossés-limites de la bande de terre permettrait de vérifier si cet espace de $40 \mathrm{~m}$ correspond à un défrichement forestier ou uniquement à la délimitation d'un espace public. La question est la même entre Escles et Portieux. Un relevé LiDAR complémentaire pourrait révéler si les régions déjà défrichées au moment de la création de cet axe sont traversées ou non par cette bande de terrain et si les deux parcours de portage, pédestre et routier, sont également décelables.

Enfin, il faudra s'interroger sur la datation et le phasage de l'ensemble de ces aménagements ainsi que sur l'évolution de l'utilisation de ces itinéraires. La voie de Corre à Escles n'a pas généré de ville. Le commerce devait donc y être limité. Il a même peut-être été délaissé ${ }^{13}$ précocement au profit d'itinéraires plus intéressants (Pasquini \& Petit 2016). La réalisation de fouilles archéologiques de cette bande de terre et de cette chaussée - qui ont pu toutes deux être des voies de portage - à divers endroits préservés entre Corre et Escles permettront, à l'aide de coupes transversales, de préciser les caractéristiques de ces aménagements et d'essayer de vérifier si ces espaces ont été défrichés. Dans un deuxième temps, un relevé LiDAR entre Escles et Portieux pourra peut-être dévoiler l'intégralité de ce parcours idéal entre la Saône et la Moselle.

\section{Bibliographie}

Bertin, O., P. Fetet et J.-J. Gaffiot 1997. «La voie de Corre à Charmes », in « Rapport de prospection sur le canton de Darney », Association Escles-Archéologie, Épinal : 2-11.

Bertin, O., P. Fetet et J.-J. Gaffiot 1998. «Voie de Saône à Moselle », in « Rapport de prospection sur le canton de Darney », Association Escles-Archéologie, Épinal : 22-28.

Bertin, O., P. Fetet et J.-J. Gaffiot 1999. «Le village antique s'agrandit» et «Structure en bois à Portieux », in « Rapport de prospection sur le canton de Darney », Association Escles-Archéologie, Épinal : 36-43 et 71-72.

Bertin, O., P. Fetet et J.-J. Gaffiot 2001. «À la recherche de la voie de Saône à Moselle », in « Rapport de prospection archéologique », Association Escles-Archéologie, Épinal : 16-18.

Bertin, O., P. Fetet et J.-J. Gaffiot 2003. «À la recherche de la voie de Saône à Moselle », in « Rapport de prospection archéologique », Association Escles-Archéologie, Épinal : 5-8.

Bertin, O., P. Fetet et J.-J. Gaffiot 2005. «La chapelle Saint-Valbert», in «Rapport de prospection archéologique », Association Escles-Archéologie, Épinal : 21-25.

Bichet, V., A. Barbier, V. Chevassu, D. Daval, É. Gauthier, M. Montandon, H. Richard et M. Thivet 2019. «Traverser les montagnes du Jura: identification de voies antiques de franchissement de la haute chaîne jurassienne par analyse LiDAR », in N. Lemaitre (dir.), Des routes et des hommes : la construction des échanges par les itinéraires et les transports, Actes des congrès nationaux des sociétés historiques et scientifiques (édition électronique) : 27-32.

Coulon, G. 2007. Les voies romaines en Gaule, Éditions Errance, Collection Promenades archéologiques, Paris.

Faure-Brac, O. 2002. La Haute-Saône, Carte archéologique de la Gaule, Académie des Inscriptions et Belles-Lettres, Paris.

Fetet, P. 2005. «Escles, ville gallo-romaine?», Annales de la Société d'Émulation du département des Vosges, $16: 61$ 75.

Fetet, P. 2009a. «La voie de Corre à Escles », in Michel J.-F. (dir.), La forêt de Darney. Des arbres et des hommes, Éditions Dominique Guéniot et Association Saône Lorraine, Langres : 101-104.

\footnotetext{
${ }^{12}$ Cette voie antique, reconnue sur $18 \mathrm{~km}$, semble relier deux axes : la voie de Corre à Escles et la voie de Langres à Portieux. II est possible également qu'elle ait pu se poursuivre au nord vers Toul et soit ainsi elle-même une section d'une autre voie de portage, postérieure à celle étudiée dans cet article, reliant la Saône et la Moselle depuis des ports qui peuvent accueillir de plus gros bateaux.

${ }^{13}$ Les causes originales de cet abandon hypothétique peuvent être multiples, mais on doit d'abord penser à des raisons purement physiques : ensablement des petites rivières, sécheresses. Le barrage sur le Coney aux Voivres (88), ouvrage abandonné en cours de construction, pourrait par exemple être interprété comme un projet de réservoir pour réguler le débit de la Saône à Corre (Fetet 2018)
} 
Fetet, P. 2009b. «L'occupation du sol et le peuplement de la Vôge (Vosges et Haute-Saône) dans l'Antiquité », thèse de doctorat, sous la direction de Y. Burnand et C. Abadie-Reynal, soutenue le 10 octobre 2009, Nancy, université de Nancy 2, 2 vol.

Fetet, P. 2017. «La voie romaine de Monthureux-sur-Saône et son environnement », in J.-P. Rothiot, J.-P. Husson et P. Labrude (dir.), De l'Ourche à la Sâ̂ne entre Clairey et Monthureux, Actes des $18^{\mathrm{e}}$ Journées d'études vosgiennes des 20 au 23 octobre 2016, Fédération des sociétés savantes des Vosges, Saône Lorraine : 43-58.

Fetet, P. 2018. "L'ouvrage gallo-romain du Pont des Fées», in Boulanger, K., Moulis, C. (dir.), La pierre dans l'Antiquité et au Moyen Âge en Lorraine. De l'extraction à la mise en æuvre, Presses universitaires de Nancy Éditions universitaires de Lorraine, Nancy : 307-316.

Fetet, P. et J-J. Gaffiot 2016. "Étude d'un itinéraire antique dans la forêt de Darney à partir du LiDAR », Annales de la Société d'Émulation du département des Vosges, $26: 35-40$.

Feugère M. et L. Bonnamour 1996. «Les armes de la Saône», in M. Reddé (dir.), L'armée romaine en Gaule, Paris : 138-146.

Gaffiot, J.J. 2001. «Un site gallo-romain réoccupé à l'époque moderne. Vioménil. Forêt de "La Pille”, D.F.S. Fouille programmée », Association Escles-Archéologie, Épinal.

Kerrio, J.-P. 2019. «Colonne au dieu cavalier», L'Écho des 3 Provinces, 198, février-mars 2019 : 6-7.

Ladaique, G. 2009. «Verrières et verreries de 1369 à 1789 », in J.-F. Michel (dir.), La forêt de Darney hier et aujourd'hui, Éditions Dominique Guéniot et Association Saône Lorraine, Langres : 193-

Mangin, Ch.-L. 1821. «Premier essai sur les chemins anciens qui traversaient le canton de Darney en Vosges », Mémoires rédigés par les membres de la Commission des Antiquités du département des Vosges, vol. 3, p. 228-260, Arch. Dép. Vosges : 9 T 14.

Mangin, M. et N. Bonvallot 2002. "Les agglomérations secondaires de Haute-Saône», in O. Faure-Brac, La HauteSaône, Carte archéologique de la Gaule, Académie des Inscriptions et Belles-Lettres, Paris : 67-68.

Pasquini, B. et Ch. Petit 2016. "Le portage entre la Saône et la Moselle dans l'Antiquité (I ${ }^{\mathrm{er}}-\mathrm{IV}^{\mathrm{e}}$ siècles). Recherches d'itinéraires », in F. Giligny et Z. Tsirtsoni (dir), Les nouvelles de l'archéologie, 142, Environnement et société : 2732. 